\title{
I dosos vacinados e não vacinados contra a influenza: morbidade relatada e aspectos sociodemográficos, Porto Alegre (RS, Brasil), 2004
}

\author{
Influenza-vaccinated and non-vaccinated elderly: \\ reported morbidity and sociodemographic aspects, \\ Porto Alegre (RS, Brazil), 2004
}

M aria A parecida M üller Vilarino ${ }^{1}$

M arta Júlia M arques Lopes ${ }^{2}$

AndréLuís M achado Bueno ${ }^{2}$

Maria Regina Varnieri Brito ${ }^{1}$

${ }^{1}$ Secretaria M unicipal de Saúde de Porto Alegre. Av. João Pessoa 325.

Farroupilha 90040-000

Porto Alegre RS.

mavilarino@

sms.prefpoa.com.br

${ }^{2}$ Escola de Enfermagem,

UniversidadeFederal do

Rio Grande do Sul.
Abstract This descriptive transversal epidemiological study had the objective of comparing the elderly population who took the influenza vaccine and who did not regarding the occurrence of events of diseases or hospital admittances within three months after the vaccination. It was not possible to work with probable sampling and the attempt of pairing the vaccinated and non-vaccinated elderly was not successful due to the high vaccine coverage observed ( $73 \%$ of the target population) and due to the short time available to make the interviews. The result of the descriptive analysis of the 1,130 elderly interviewed was quite interesting even not being possible to infer it regarding the universe of the elderly population from Porto Alegre. We found a higher proportion of vaccinated people in the age group of 70 to 79 years old ( $42 \%)$, and a prevalence of non-vaccinated among the age group of 60 to 64 years old (40\%). The vaccinated el derly were mostly older; female, who have private health care insurance; with higher income; that perform physical activities and non-smokers. The non-vaccinated were mostly men; younger; with lower income; that do not perform physical exercises; and smoke. A lower percentage of pneumonias reports and hospital admittances was observed among the vaccinated in comparison to the non-vaccinated people. Key words Elderly, Vaccination, Influenzae
Resumo Aproveitando o evento da campanha nacional de vacinação de idosos contra o vírus influenza em 2004, realizou-se um estudo epidemiológico transversal descritivo com objetivo de comparar populações de idosos vacinadas e não vacinadas em relação à ocorrência de situações de doença ou internações hospitalares dentro do período de três meses após a vacinação. N ão foi possível trabalhar com amostragem probabilística, e a tentativa de pareamento de idosos vacinados e não vacinados não teve sucesso devido à elevada cobertura vacinal observada ( $73 \%$ da populaçãoalvo) e ao curto espaço de tempo para obter as entrevistas. 0 resultado da análise descritiva dos 1.130 idosos entrevistados foi muito interessante, mesmo não sendo possível inferir para o universo de idosos de Porto Alegre. Encontrou-se maior proporção na população vacinada de pessoas na faixa etária de 70 a 79 anos ( $42 \%$ ) ena população não vacinada na faixa de 60 a 64 anos (40\%). Os idosos vacinados são na maioria mais velhos; mulheres; têm plano de saúde; declaram renda mais elevada; realizam atividades físicas e não são fumantes. Os idosos não vacinados são na maioria homens; mais jovens; com menor renda; não realizam atividades físicas e são fumantes. O bservou-se percentual menor de relato de pneumonias e internações hospitalares entre os vacinados em relação aos não vacinados. Palavras-chave Idosos, Vacinação, Influenza 


\section{Introdução}

Este estudo tem como base a dissertação de mestrado intitulada A (re) volta da vacina: eficácia e credi bilidade social da vacinação contra influenza entre idosos de Porto Alegre. N ela identificou-se, através de uma sériehistórica deinternações hospitalares e óbitos por pneumonias, uma tendência à queda desses eventos a partir do início das Campanhas de Vacinação contra Influenza entre Idosos no Município de Porto Alegre, RS ${ }^{1}$.

Com base nesses resultados surgiu a ideia de se investigar seexiste relação entre o uso da vacina contra influenza e as internações hospitalares por pneumonias em um grupo de idosos de Porto Alegre, considerando um determinado período de tempo, comparando um grupo de idosos vacinados com outro grupo de idosos que não houvesse recebido a vacina.

A construção do problema de estudo baseouse nas modificações das políticas de saúde ocorridas no país nas últimas décadas. Destacou-se, como dado fundamental, a mudança ocorrida no perfil demográfico brasileiro, que, em decorrência da diminuição da fecundidade e da mortalidade, apresenta um aumento da população de idosos. Essa nova parcela da população demanda do setor público a implantação de políticas que atendam às suas necessidades específicas. Para os indivíduos de sessenta anos de idade ou mais, quecorrespondem ao grupo etário mais representativo para doenças crônicas e de crescimento mais rápido na população do país, a influenza e suas complicações permanecem como uma causa importante de morbimortalidade 2 .

A prevenção mais eficaz para o vírus influenza, a qual écapaz de reduzir sua incidência eseveridade, éa vacinação contra a gripe. A vacinação é um procedimento recomendado pela Organização M undial da Saúde(OM S) e beneficia tanto indivíduos sadios como os mais suscetíveisa episódios gripais, nos quais se incluem portadores de doenças crônicas pulmonares, cardiovasculares, metabólicas e imunodeprimidos. A gripe pode agravar a patologia básica de indivíduos com distúrbios cardíacos e/ou pulmonares. Essas complicações são causa frequente de hospitalizações prolongadas e de aumento do número de mortes por pneumonia e/ou patologias subjacentes, vinculadas aos surtos gripais que ocorrem anualmente. A vacinação deindivíduos com alto risco está associada a uma diminuição significativa da gravidade da doença em relação à incidência dehospitalizações emortalidadeassociadas à gripe. Nos países em que as quatro estações do ano são mais marcadas, as epidemias de gripe ocorrem virtualmente a cada inverno. Durante um surto típico nos Estados Unidos, aproximadamente $30 \%$ da população ( 70 milhões) tornam-se enfermos. D esses, 400.000 são hospitalizadose entre 10.000 e 20.000 morrem em consequência de complicações. Embora seja considerada uma enfermidade benigna e autolimitada, o estado gripal nos idosos apresenta maior importância epidemiológica, em face desuasconsequências, aumento de gastos hospitalares e mortalidade. Além disso, o idoso com uma infecção por influenza apresenta maior predisposição para desenvolver pneumonia ${ }^{3}$.

Estudos do Centers for Disease Control and Prevention (CDC) mostram que a vacina reduz a hospitalização em cerca de $70 \%$ e os óbitos em cerca de $85 \%$. Em casas geriátricas, a vacina pode reduzir o risco de hospitalização em aproximadamente $50 \%$, a incidência de pneumonias em cerca de $60 \%$ e o risco de morrer de $75 \%$ a $80 \%$. Quando ocorre a circulação de um vírus diferente dos contemplados na composição da vacina, a sua eficácia pode ser menor em relação à incidência das infecções, mas a vacina poderá ainda diminuir a severidadeda infecção e prevenir complicações ea morte 4 .

Outro estudo realizado nos Estados Unidos durantetrês estações deinfluenza, 1989-90, 199192, 1992-93, no qual, a cada ano, foram incluídos mais de 25.000 idosos com 65 anos e mais, mostrou que em geral a vacinação diminuiu em $48 \%$ a $57 \%$ as admissões hospitalares por pneumonias e influenza. Mais importante ainda, a vacinação foi associada a uma diminuição de $39 \%$ a $54 \%$ da mortalidade, considerando todas as causas durante os três períodos do estudo ${ }^{5}$.

Em Porto Alegre, verificou-se uma redução de 25,2\% nas internações hospitalares por pneumonia no período de 1996 a 2000. No mesmo período, houve uma diminuição de $34,3 \%$ na mortalidade por pneumonias 1 . A inclusão da vacina contra influenza no Calendário Nacional de Imunizações no Brasil inspirou-se nas iniciativas do município edo estado de São Paulo, que tornaram lei a vacinação de idosos contra a gripe. Editadas em 1997 e 1998, respectivamente, ambas as leis originaram-se na experiência do Centro de Estudos do Envelhecimento da Universidade Federal de São Paulo ${ }^{6}$. N essa perspectiva, a partir de 1999 o M inistério da Saúde(M S) deu início a uma nova estratégia na prática da vacinação, quefoi a edição da campanha devacinação contra influenza em todo o território nacional, com o objetivo de vacinar a população acima de 65 anos. Essa prática visa, portanto, diminuir as complicações e internações advindas 
da doença influenza, popularmente denominada gripe- mais especificamente, as pneumonias, que ocorrem com maior freqüência nessa faixa etária mais vulnerável ${ }^{7}$.

Esse novo panorama estimulou a produção de outros estudos locais que pudessem dar continuidade e sustentação às tendências observadas. Com isso, uma nova estratégia de promoção da saúde e, portanto, um novo desafio para os trabalhadores da Saúde Coletiva estavam se delineando. Algumas reflexões foram se desenhando no cotidiano de nossa prática, no Núcleo de Imunização da Secretaria Municipal de Saúde de Porto Alegreeno Grupo deEstudosem Saúde Coletiva (GESC) da Escola de Enfermagem (EENF) da Universidade Federal do Rio Grande do Sul (UFRGS), no que se refere a essa vacinação destinada aos idosos, as quais deram origem à elaboração de problemas de pesquisa, conduzindo-nos ao encaminhamento das questões deste estudo.

\section{Objetivos}

Este estudo teve como objetivos:

- Investigar a relação entre a vacina contra influenza e a ocorrência de pneumonias e de internações hospitalares em um grupo de idosos de Porto Alegre.

- Comparar um grupo de idosos vacinados com outro grupo que não recebeu a vacina.

- Caracterizar esses idosos quanto a aspectos sociodemográficos no sentido de conhecer hábitos devida e saúdeque influenciam esse processo específico deadoecimento.

\section{M etodologia e coleta de dados}

0 desenho de pesquisa inicial previa um estudo de caso-controle pareado utilizan do idosos vacinados entrevistados em um centro de saúde localizado na cidade de Porto Alegre, com grande afluxo de pessoas durante todas as campanhas de vacinação. 0 grupo controle seria buscado através da entrevista de idosos que procurassem o mesmo serviço por outros motivos e idosos moradores da região por meio de visitas domiciliares - é uma das regiões de Porto Alegre com maior população proporcional de idosos.

Calculou-se a necessidade de 565 sujeitos necessários em cada um dos grupos para, com poder do testede $80 \%$ esignificância de $5 \%$, encontrar uma proteção de $60 \%$. Utilizou-se uma incidência estimada de $10,61 \%$ deinternações hospi- talares por pneumonias, por mil habitantes, observado no ano de 1995, antes da introdução da vacina contrainfluenza, euma incidência de 6,04\% de internações hospitalares por pneumonias, por mil habitantes no ano de 2001, observada após a introdução da vacina contra influenza?

0 período de acompanhamento foi definido considerando-se que um pico máximo de anticorpos contra o vírus influenza ocorre após quatro a seis semanas da aplicação da vacina, embora em idosos os níveis de anticorpos possam ser menores, o que justificou o tempo de três meses (12 semanas) de efeito protetor causado pela vacina.

Conformea Resolução n ${ }^{\circ} 196$, de 10 de outubro de 1996, do Consel ho N acional de Saúde 8 , 0 estudo constitui-se em uma pesquisa que envolve seres humanose, portanto, atendeu às exigências éticas e científicas fundamentais. Os aspectos éticos da pesquisa implicam o consentimento livree esclarecido dos indivíduos-alvo ea proteção a grupos vulneráveis e aos legalmente incapazes; o compromisso de oferecer o máximo de benefícios e o mínimo de danos e riscos; a relevância social da pesquisa com vantagens significativas aos sujeitos do estudo. 0 projeto de pesquisa foi encaminhado ao Comitê de Ética da Secretaria Municipal de Saúde de Porto Alegre, sendo apreciado e aprovado.

Para respeitar esses princípios, os idosos foram esclarecidos cuidadosamente sobre os objetivos da pesquisa e dos procedimentos realizados. Em seguida, foi apresentado um Termo de Consentimento Livreel nformado, no qual constaram a assinatura do entrevistado mediante a ciência do conteúdo do documento esua autorização para a posterior visita domiciliar . Foram também obedecidos os princípios éticos em relação ao acesso e análise dos dados, respeitando as normas de pesquisa em saúde referidas.

$\mathrm{Na}$ execução da fase de coleta de dados, foram enfrentadas dificuldades operacionais: primeiramente, a falta definanciamento para a pesquisa, o que levou a necessi dade de realização do trabalho de campo por voluntários. 0 número de voluntários foi expressivo (estudantes do Curso de Enfermagem da UFRGS) para o início do projeto. No entanto, atividades acadêmicas acarretaram muitas desistências ao longo do trabal ho. A coleta teveque ser completada por estagiários da Equipe de Vigilância das Doenças Transmissíveis (EVDT) da Coordenadoria Geral de Vigilância em Saúde(CGVS) da Secretaria Municipal de Saúde de Porto Alegre.

A busca inicial dos indivíduos vacinados realizou-se no Centro de Saúde "M odelo", com o 
objetivo de obter os 565 idosos (60 e mais anos) vacinados preconizados na amostra, sem quase nenhum problema operacional, pois o afluxo de pessoas era grande durante a campanha de vacinação e os alunos voluntários haviam sido re centemente incorporados ao grupo de trabal ho.

Após os três meses da aplicação da vacina, realizaram-se visitas domiciliares para a coleta dos dados de acompanhamento do período. Também foi utilizada a entrevista por telefone aos idosos inicial mente entrevistados no serviço de saúde.

Para a obtenção das 565 pessoas não vacinadas (grupo controle pareado), optou-se, após tentativas fracassadas de descobrir contatos por indicações dos vacinados e vizinhança de en dereço como constava do projeto original, por realizar as entrevistas no M ercado Público de Porto Alegre (mesma região da cidade do posto de saúde, cerca de dois quilômetros de distância), onde circulam milhares de pessoas diariamente. A pós um mês de entrevistas no M ercado Público, obtevese então o grupo dos não vacinados. 0 problema maior não foi a recusa, esim a carência deidosos não vacinados queestivessem em seus domicílios em horário comercial. Com uma cobertura vacinal elevada ( $72,74 \%$ da população total com 60 e mais anos residente em Porto Alegre), restaram poucos idosos não vacinados na cidade e ainda dispersos em um território de 496 quilômetros quadrados. Não foi utilizada a visita a clínicas geriátricas para entrevistar tanto vacinados quanto não vacinados, pois a epidemiologia da gripeé diferente na população não institucionalizada.

As entrevistas dos idosos vacinados ocorre ram em dois momentos: no dia da aplicação da vacina e três meses depois, em visita domiciliar ou contato telefônico. A entrevista dos idosos não vacinados, no mesmo período de tempo de trtês meses após a campan ha de vacinação, ocorreu em um só momento: ou em visita domiciliar ou no momento da entrevista no M ercado Público de Porto Alegre.

$\mathrm{N}$ ão foi possível obter dados pareados entre os entrevistados com relação a idade, sexo, renda enível educacional. A falta decomparabilidade entre os grupos levou à limitação das análises em sua maioria ao nível descritivo. M esmo com essas limitações, dados importantes emergiram da pesquisa, os quais poderão contribuir para 0 trabal ho realizado nos serviços de saúde eno planejamento de ações de saúde. 0 prolongamento indefinido do tempo de coleta para conseguir idosos não vacinados pareados com os vacinados inviabilizaria o tempo de observação de três meses para patologias e internação hospitalar; nem mesmo a análise epidemiológica descritiva seria possível.

Tínhamos então dois grupos de idosos, não pareados, de seleção não aleatória, observados no mesmo período de tempo (segundo semestre de 2004), com a aplicação de questionários de perguntas retrospectivas sobre doenças e internações hospital ares. Portanto, esteestudo passou a ser um estudo epidemiológico transversal descritivo, que não permite inferir seus resultados para a população idosa residente em Porto Alegre.

Foi utilizado o software livre EPI-Info para a criação do banco de dados e para a análise estatística dos dados.

\section{Resultadosediscussão}

Ao analisarmos a distribuição etária de nossos grupos de idosos vacinados e não vacinados, percebemos que ela difere da população de idosos de Porto Alegre (Tabela 1).

Essa diferença sugere que a população menos idosa, portanto mais ativa e provavelmente menos doente, entrevistada em sua maior parte na área do M ercado Público municipal, não atendeu como esperado ao chamado para a vacinação. Já a população vacinada, entrevistada em um centro de saúde, basicamentemais idosa etal vez com maior número de doenças crônicas, no entanto buscou espontaneamentea vacina. Devemos lembrar que os indivíduos institucionalizados (abrigos, clínicas, hospitais) foram vacinados em suas instituições enão foram incluídos nesta pesquisa. Esses idosos vacinados compareceram ao centro de saúde, portanto têm também mobilidade. Infelizmente, o questionário não incluía a informação sobre o idoso necessitar de ajuda para se locomover. Aqui observamos que um dos grandes objetivos da vacinação foi alcançado: vacinar os mais idosos e os mais doentes, pois são a população mais beneficiada pela vacina na prevenção da morbimortalidade desencadeada pela infecção pelo vírus influenza.

Em relação à distribuição por sexo, observamos que ela não é reproduzida em nenhum dos dois grupos estudados. Na população geral de Porto Alegre, na faixa etária de 60 e mais anos, temos, em 2004, 37\% dehomens e $63 \%$ demulheres. No grupo de idosos vacinados, encontramos $43 \%$ de homens e $57 \%$ de mulheres; já no grupo de idosos não vacinados encontramos 50,45\% de homens e 49,55\% de mulheres. Essa diferença foi causada pelo desenho inicial da pesquisa, que buscava incluir em cada grupo $50 \%$ de idosos de cada sexo. N ovamente, a dificuldade encontrada 
Tabela 1. Distribuição por faixa etária dos idosos. População residente, grupo de vacinados e grupo de não vacinados, Porto Alegre, 2004.

\begin{tabular}{|c|c|c|c|c|c|}
\hline & \multicolumn{5}{|c|}{ Faixas etárias } \\
\hline & $\begin{array}{l}60-64 \\
\text { anos }\end{array}$ & $\begin{array}{l}65-69 \\
\text { anos }\end{array}$ & $\begin{array}{c}70-79 \\
\text { anos }\end{array}$ & $\begin{array}{l}80 \mathrm{e}+ \\
\text { anos }\end{array}$ & total \\
\hline $\begin{array}{l}\text { População de Porto Alegre } 2004 \text { - Datasus } \\
\text { ambos os sexos } \\
\text { masculina } \\
\% \\
\text { feminina } \\
\%\end{array}$ & $\begin{array}{r}48.374 \\
20.025 \\
32,48 \\
28.349 \\
27,23\end{array}$ & $\begin{array}{r}41.450 \\
16.293 \\
26,43 \\
25.157 \\
24,17\end{array}$ & $\begin{array}{r}54.677 \\
19.526 \\
31,67 \\
35.151 \\
33,77\end{array}$ & $\begin{array}{r}21.242 \\
5.806 \\
9,42 \\
15.436 \\
14,83\end{array}$ & $\begin{array}{r}165.743 \\
61.650 \\
100,00 \\
104.093 \\
100,00\end{array}$ \\
\hline $\begin{array}{l}\text { População de idosos da amostra de vacinados* } \\
\text { masculina } \\
\% \\
\text { feminina } \\
\%\end{array}$ & $\begin{array}{r}51 \\
21,16 \\
60 \\
18,69\end{array}$ & $\begin{array}{r}64 \\
26,56 \\
75 \\
23,36\end{array}$ & $\begin{array}{r}103 \\
42,74 \\
133 \\
41,43\end{array}$ & $\begin{array}{r}23 \\
9,54 \\
53 \\
16,51\end{array}$ & $\begin{array}{r}241 \\
100,00 \\
321 \\
100,00\end{array}$ \\
\hline $\begin{array}{l}\text { População de idosos da amostra de não vacinados* } \\
\text { masculina } \\
\% \\
\text { feminina } \\
\%\end{array}$ & $\begin{array}{r}108 \\
38,71 \\
118 \\
43,07\end{array}$ & $\begin{array}{r}79 \\
28,32 \\
65 \\
23,72\end{array}$ & $\begin{array}{r}71 \\
25,45 \\
74 \\
27,01\end{array}$ & $\begin{array}{r}21 \\
7,53 \\
17 \\
6,20\end{array}$ & $\begin{array}{r}279 \\
100,00 \\
274 \\
100,00\end{array}$ \\
\hline
\end{tabular}

*Excluídos os com sexo ou faixa etária ignorados.

no trabalho de campo sugere que foi mais fácil encontrar idosas no serviço de saúde e idosos no Mercado Público. Aqui, nessa variável "sexo", o viés de seleção foi muito importante (Tabela 2).

Observa-se que os idosos vacinados apresentam uma distribuição de renda maior do que os idosos não vacinados. I sto tanto pode sugerir que a população de idosos de menor poder aquisitivo, portanto maior vulnerabilidade social, não tem procurado a vacinação, quanto pode sugerir que essa população não frequentaria os serviços de saúde de atenção básica onde foram entrevistados os vacinados. Esse mesmo grupo de idosos não vacinados relata que procura, sim, esses serviços ao ficar doente (42\%), o quesugere que eles não procuram a vacinação porque não valorizam essa ação. Já a população vacinada, entrevistada dentro do serviço de saúde público, procura o serviço privado ou conveniado ao sesentir doente (64\%). Considerando que os indivíduos vacinados foram captados na rede pública e os não vacinados fora dos serviços públicos de saúde, esperava-se um viés de seleção de direção oposta.

Reforçando a postura de autocuidado, ou de preservação da própria saúde, da população de idosos do grupo vacinado, vemos que a proporção defumantesébem mais baixa entre os vacinados ( $11 \%)$, contra $31 \%$ do grupo de idosos não vacinados, assim como a prática de atividades físicas é maior no grupo vacinado, mesmo que eles tenham se autodeclarado "doentes", antes e de- pois da vacinação, em maior proporção do que os idosos não vacinados. Os idosos do grupo não vacinado são mais sedentários e relatam ser menos doentes e ter adoecido menos nos últimos três meses em relação ao grupo vacinado.

Ao analisarmos as informações sobre as doenças anteriores à vacinação relatadas pelos dois grupos, observamos que praticamente $50 \%$ do grupo de idosos vacinados relatou apresentar doenças do aparel ho cardiovascular (principalmente hipertensão arterial sistêmica ecardiopatias isquêmicas). Já no grupo não vacinado, essa proporção foi bem menor (37\%), sendo compatível com a ausência de sintomas clínicos específicos dessas doenças em sua fase inicial. A maior proporção de internações por doenças cardiovasculares no grupo não vacinado no período de estudo sugere que essetipo deproblema crônico de saúde poderia ser desconhecido desses idosos. A bibliografia sobre o tema sugere que tanto para hipertensos quanto para os portadores de doenças cardíacas a vacina da gripe reduz complicações e consequentes internações hospitalares, o que beneficia substancialmente o grupo de idosos vacinados.

Outro aspecto que podemos observar é a maior proporção de idosos que negam qualquer tipo de doença no grupo não vacinado (32\%) em relação ao grupo vacinado (24\%).

Aqui, com certeza, temos diferenças de percepções desses dois grupos de idosos (vacinados e não vacinados) sobre ser ou estar doente. Para 
Tabela 2. D istribuição de algumas variáveis do questionário da pesquisa entre os grupos vacinados e não vacinados, por sexo. Porto Alegre, 2004.

\begin{tabular}{|c|c|c|c|c|}
\hline Variável do questionário & Vacinados & $\%$ & $\mathrm{~N}$ ão vacinados & $\%$ \\
\hline \multicolumn{5}{|l|}{ Renda } \\
\hline$<R \$ 500$ & 101 & 17,88 & 85 & 15,04 \\
\hline $\mathrm{R} \$ 500$ a R\$ 999 & 124 & 21,95 & 187 & 33,10 \\
\hline $\mathrm{R} \$ 1.000$ a $\mathrm{R} \$ 1.999$ & 122 & 21,59 & 113 & 20,00 \\
\hline $\mathrm{R} \$ 2.000$ ou mais & 148 & 26,19 & 83 & 14,69 \\
\hline Não declarado & 70 & 12,39 & 97 & 17,17 \\
\hline Somatório & 565 & 100 & 565 & 100 \\
\hline \multicolumn{5}{|l|}{ Quando adoece, procura: } \\
\hline Serviço público - posto de saúde & 117 & 20,71 & 236 & 41,77 \\
\hline Serviço privado ou convênio & 361 & 63,89 & 207 & 36,64 \\
\hline Outra resposta (inclui emer. hospitalar) & 87 & 15,40 & 122 & 21,59 \\
\hline Somatório & 565 & 100 & 565 & 100 \\
\hline \multicolumn{5}{|l|}{ Tabagismo } \\
\hline Fumante & 62 & 10,97 & 174 & 30,80 \\
\hline Não fumante & 503 & 89,03 & 391 & 69,20 \\
\hline Somatório & 565 & 100 & 565 & 100 \\
\hline \multicolumn{5}{|l|}{ Atividade física } \\
\hline Nenhuma & 192 & 33,98 & 252 & 44,60 \\
\hline Caminhadas & 302 & 53,45 & 233 & 41,24 \\
\hline Outras & 62 & 10,97 & 64 & 11,33 \\
\hline Ignorado & 9 & 1,59 & 16 & 2,83 \\
\hline Somatório & 565 & 100 & 565 & 100 \\
\hline \multicolumn{5}{|l|}{ Problemas de saúde prévios à vacinação relatados } \\
\hline Nega doenças & 133 & 23,54 & 183 & 32,39 \\
\hline Doenças do Sistema Endócrino & 61 & 10,80 & 74 & 13,10 \\
\hline Doenças do Sistema Cardiovascular & 282 & 49,91 & 207 & 36,64 \\
\hline Doenças Osteomusculares & 89 & 15,75 & 101 & 17,88 \\
\hline Somatório & 565 & 100 & 565 & 100 \\
\hline \multicolumn{5}{|l|}{ Esteve doente nos últimos 3 meses? } \\
\hline Sim & 170 & 30,09 & 112 & 19,82 \\
\hline Não & 395 & 69,91 & 453 & 80,18 \\
\hline Somatório & 565 & 100 & 565 & 100 \\
\hline \multicolumn{5}{|l|}{ Se Sim na pergunta anterior, qual a doença } \\
\hline Problema cardíaco & 5 & 2,94 & 10 & 8,93 \\
\hline Problema respiratório (exclui pneumonia) & 147 & 86,47 & 72 & 64,29 \\
\hline Pneumonia & 1 & 0,59 & 4 & 3,57 \\
\hline Outros (inclui câncer) & 16 & 9,41 & 24 & 21,43 \\
\hline Ignorado & 1 & 0,59 & 2 & 1,79 \\
\hline Somatório & 170 & 100 & 112 & 100 \\
\hline
\end{tabular}

*Excluídos os com sexo ou faixa etária ignorados.

147 idosos vacinados que referiram doenças do aparelho respiratório, temos somente um que relatou ter tido pneumonia. Já no grupo de idosos não vacinados, para 72 que referiram doenças do aparelho respiratório, temos quatro que relataram ter tido pneumonia - razões de 1/147 e 4/72 ( ou 1/18, um em cada 18). Aqui, mesmo com viés de seleção importante, devemos lembrar que os idosos vacinados foram entrevistados dentro de um serviço de saúde eem seus domićlios, en- quanto os não vacinados foram entrevistados"na rua" em uma área de comércio, implicando que os idosos não vacinados que faleceram ou estivessem muito doentes não estariam disponíveis para serem entrevistados, gerando um viés em sentido inverso às razões observadas.

Se ignorarmos o viés de sel eção deste estudo e utilizarmos o Statcalc do EPI6, encontramos um teste exato de Fisher com $p<0,046$ ao compararmos estas duas razões (doença respirató- 
ria x pneumonia). Portanto, apesar de todos os problemas metodológicos, a vacinação contra a influenza mostrou-se protetora para a ocorrência de pneumonia na população de idosos.

Se compararmos a ocorrência de problemas cardíacos, no grupo devacinadosencontramoscinco casos em 170 doentes $(2,9 \%)$, contra os dez casos em 112 doentes (8,9\%) no grupo de não vacinados, existindo a possibilidade de um efeito protetor da vacina. No entanto, para esse tipo de análise os vieses são diversos e em sentidos diversos, pois a relação entre doença cardíaca e vacinação para uma infecção respiratória é mais complexa e de efeito indireto. Aqui, as razões de 5/165 (ou 1/ 33) no grupo de idosos vacinados, e de 10/102 (1/ 10,2 ) no grupo não vacinado, se tabuladas $2 \times 2$, apresentam uma razão de riscos de 0,31 (cerca de $30 \%$ de proteção da vacina para doença cardiovascular), prejudicada esta relação por um intervalo de confiança de $95 \%$ variando de 0,09 a 1,02.

$\mathrm{Na}$ Tabela 3, observamos que, apesar de 0 grupo vacinado ser mais velho, relatar mais doenças antes e depois da aplicação da vacina e ser captado dentro de um serviço de saúde, mesmo assim a vacinação mostrou-se indiscutivelmente com efeito protetor para internações hospitalares em geral. Aqui, todos os vieses de sel eção do estudo são contrários ao efeito protetor da vacina.

$\mathrm{Na}$ Tabela 4, podemos observar os motivos relatados pelos idosos para essas internações. 0 que merece maiores estudos é que essas internações não ocorreram pelos motivos esperados pe los pesquisadores (doenças respiratórias), e sim por diversas outras causas. Tais internações não foram verificadas em prontuários médicos ou outrasfontes deinformação, sendo exclusivamente baseadas nos relatos dos entrevistados. D evido aos baixos valores absolutos observados, não foi utilizada análise estatística nos dados da Tabela 4.

Não houve óbitos no grupo vacinado no pe ríodo de tempo observado na pesquisa. No grupo não vacinado, a metodologia utilizada não detecta a ocorrência de óbitos.

Tabela 3. Internações hospitalares relatadas no período de até três meses após a campanha de vacinação contra influenza. Porto Alegre, 2004.

\begin{tabular}{lccr}
\hline & Internaram & $\begin{array}{c}\text { Não } \\
\text { internaram }\end{array}$ \\
\hline Vacinados & 9 & 556 & 565 \\
Não vacinados & 26 & 539 & 565 \\
& 35 & 1.095 & 1.130 \\
\hline
\end{tabular}

Razão de riscos de 0,34 (IC95\% de 0,14 a 0,76).
O questionário aplicado também apresentava uma questão aberta sobre os motivos que levaram os idosos a procurar ou não a vacina contra a influenza. Podemos observar esses resultados na Tabela 5 e utilizá-los para qualificar as ações de divulgação das campanhas de vacinação com o objetivo de ampliar a população vacinada.

Tabela 4. M otivos de internações hospitalares relatados, idosos vacinados e não vacinados contra influenza, durante os três meses após a vacinação. Porto Alegre, 2004.

\begin{tabular}{lccrr}
\hline \multicolumn{1}{c}{ Vacinado } & Sim & Não & N & $\%$ \\
\hline Hospitalização & & & & \\
Problemas cardíacos & 1 & 8 & 9 & 25,7 \\
Pneumonia & 0 & 1 & 1 & 2,9 \\
Problemas respiratórios & 3 & 1 & 4 & 11,4 \\
Outros & 5 & 16 & 21 & 60,0 \\
Total & $9(25,7 \%)$ & $26(74,3 \%)$ & 35 & 100 \\
\hline
\end{tabular}

Fonte: pesquisa direta, dados coletados no Centro de SaúdeM odelo, M ercado Público edomicílio dosidosos. Elaboração das tabelas: ALM Bueno, Porto Alegre, dezembro de 2005.

Tabela 5. Motivos de adesão e não adesão à vacinação contra influenza, segundo informações dos idosos. Porto Alegre, 2004.

\begin{tabular}{lrr}
\hline & $\mathrm{N}$ & \multicolumn{1}{c}{$\%$} \\
\hline Idosos vacinados & & \\
Prevenção da gripe & 524 & 92,74 \\
Recomendação médica & 9 & 1,59 \\
Divulgação na mídia & 3 & 0,53 \\
Não informado & 29 & 5,13 \\
Somatório & 565 & 100 \\
Idosos não vacinados & & \\
Não quis & 165 & 29,20 \\
Não acredita & 149 & 26,37 \\
Acredita que a vacina faz mal & 61 & 10,80 \\
Esqueceu & 48 & 8,50 \\
Desinformação & 12 & 2,12 \\
Estava gripado & 12 & 2,12 \\
Não fica gripado & 8 & 1,42 \\
A vacina causa gripe & 7 & 1,24 \\
Recomendação médica & 5 & 0,88 \\
Alergia & 5 & 0,88 \\
Estava doente & 4 & 0,71 \\
Tem medo & 4 & 0,71 \\
Não gosta da vacina & 2 & 0,35 \\
Não teve tempo & 1 & 0,18 \\
Ninguém levou & 1 & 0,18 \\
Não fica doente & 1 & 0,18 \\
Utiliza homeopatia & 1 & 0,18 \\
Não informado & 79 & 13,98 \\
Somatório & 565 & 100 \\
\hline & & \\
\hline
\end{tabular}




\section{Considerações finais}

Este estudo permitiu al cançar al guns resultados interessantes e destacá-los como capazes de contribuir para o aprimoramento das atividades de prevenção e educação em saúde, realizadas no âmbito dos serviços de saúde, principalmente do setor público.

Pelo exposto, o grupo dos não vacinados, que busca os serviços públicos de saúde quando necessita de assistência médica, éo mais vulnerável para o evento hospitalização. Portanto, é o grupo que merece atenção especial dos profissionais da saúde, no sentido de identificá-los e sensibilizá-los para a importância da vacinação anual contra influenza.

\section{Colaboradores}

MAM Vilarino e MJM Lopes foram responsáveis pela elaboração do projeto, em parceria com a Universidade Federal do Rio Grande do Sul ea Prefeitura de Porto Alegre, pelo desenvolvimento da pesquisa e pela redação do artigo. ALM Bueno participou do desenvolvimento da pesquisa, da coleta de dados, da estruturação do banco de dados e da redação do artigo. M RV Brito foi responsável pela análise dos dados quantitativos e também pela redação do artigo.
Pode-se sugerir que no grupo vacinado contra influenza houve uma associação positiva em relação ao uso da vacina e à prevenção de pneumonias, bem como à prevenção de internações hospitalares por todas as causas. Esses achados estão de acordo com os demais estudos que fundamentaram esta pesquisa, apesar das limitações metodológicas enfrentadas.

Dessa forma, pretende-se alertar gestores de saúde, no sentido de sensibilizá-los, e aos profissionais da saúde sobre a importância e efetividade dessa vacinação no grupo dos menos favorecidos economicamente, usuários dos serviços de atenção básica de saúde, os quais não estão esclarecidos suficientemente sobreos benefícios da vacinação contra a influenza.

\section{Referências}

1. Vilarino MAM. A (re)volta da vacina: eficácia e cre dibilidade social da vacinação contra influenza entre idosos de Porto Alegre [dissertação]. Porto Alegre: Universidade Federal do Rio Grande do Sul; 2002.

2. Toniolo Neto J. A história da gripe: a influenza em todos os tempos e agora. São Paulo: Dezembro XII Editorial; 2001.

3. Silvestre JA. Vacinação para idosos. Brasília: Ministério da Saúde; 1997.

4. Centers for Disease Control and Prevention (CDC). Prevention and control of influenza: recommendations of the Advisory Committee on Immunization Practices (ACIP). Atlanta: Morbidity and Mortality Weekly Report; 1998.

5. Nichol KL, Margolis KL, Wuorenma J, Von Sternberg $T$. The efficacy and cost effectiveness of vaccination against influenza among elderly persons living in the community. N Engl J Med 1994; 331(12):778-784.

6. Aranda C. M odelo para o mundo. Notícias VigiGripe [monografia na Internet]. 2000 [acessado $2000 \mathrm{abr}$ 12]. Disponível em: http://www.vigivirus.com.br

7. Ministério da Saúde. Informe técnico: campanha nacional de vacinação do idoso. Brasília: M inistério da Saúde; 2003.

8. Resolução $n^{\circ} 196$, de 10 de outubro de 1996. Diretrizes e normas regulamentadoras de pesquisa envolvendo seres humanos. Brasília: M inistério da Saúde; 1997.

Artigo apresentado em 24/08/2007

Aprovado em 27/11/2007

Versão final apresentada em 17/03/2008 